\title{
SOME NEW EXAMPLES OF NONORIENTABLE MINIMAL SURFACES
}

\author{
M. ELISA G. G. DE OLIVEIRA
}

\begin{abstract}
The classical Henneberg's minimal surface $(1875,[3,4,11])$ was the unique nonorientable example known until 1981, when Meeks [6] exhibited the first example of a nonorientable, regular, complete, minimal surface of finite total curvature $-6 \pi$.

In this paper, we study the nonorientable, regular, complete minimal surfaces of finite total curvature and give some examples of punctured projective planes regularly and minimally immersed in $\mathbf{R}^{3}$ and $\mathbf{R}^{n}$.
\end{abstract}

1. Nonorientable minimal surfaces in $\mathbf{R}^{n}$. We consider surfaces $S$ in $\mathbf{R}^{n}$ defined by maps $X: M \rightarrow \mathbf{R}^{n}$, where $M$ is a two-dimensional manifold.

For the study of a nonorientable, connected surface $S$ we take the double surface $\tilde{S}$ given by $\tilde{X}: \tilde{M} \rightarrow \mathbf{R}^{n}$, where $\Pi: \tilde{M} \rightarrow M$ is the oriented two-sheeted covering of $M$, and $\tilde{X}=X \circ \Pi$. We have an involution $\tilde{I}: \tilde{M} \rightarrow \tilde{M}$ without fixed points and a conformal structure on $\tilde{M}$ such that $\tilde{I}$ is antiholomorphic.

We have a representation theorem for the nonorientable minimal surfaces:

THEOREM 1.1. Let $S$ be a nonorientable regular connected minimal surface in $\mathbf{R}^{n}$. The double surface $\tilde{S}$ is a minimal surface in $\mathbf{R}^{n}$ defined by $\tilde{X}: \tilde{M} \rightarrow \mathbf{R}^{n}$,

$$
\tilde{X}(p)=\operatorname{Re} \int_{p_{0}}^{p} \tilde{\phi}(\varsigma) d \zeta, \quad p_{0}, p \in \tilde{M},
$$

with $\alpha=\tilde{\phi}(\varsigma) d \varsigma$ such that $\tilde{I}^{*} \alpha=\bar{\alpha}$ (that is, $\tilde{I}^{*} \alpha_{k}=\bar{\alpha}_{k}, 1 \leq k \leq n$ ).

Conversely, if $\tilde{S}$ is a regular orientable connected minimal surface in $\mathbf{R}^{n}$ given by (1.1) and if there is an antiholomorphic involution $\tilde{I}: \tilde{M} \rightarrow \tilde{M}$ without fixed points such that $\tilde{I}^{*} \alpha=\bar{\alpha}$, then $\tilde{S}$ is the double surface of a regular nonorientable minimal surface in $\mathbf{R}^{n}$.

The consequences of the condition $\tilde{I}^{*} \alpha=\bar{\alpha}$ with respect to the various forms of representation for minimal surfaces in $\mathbf{R}^{n}$ are

COROLlaRY 1.2 (MEEKS [6]). Let $f$ and $g$ be the functions of Weierstrass's representation of an orientable, regular, connected minimal surface $\tilde{S}$ in $\mathbf{R}^{3}$, that is

$$
\tilde{X}(p)=\operatorname{Re} \int_{p_{0}}^{p} \frac{f(\zeta)}{2}\left(1-g^{2}(\zeta), i\left(1+g^{2}(\zeta)\right), 2 g(\varsigma)\right) d \varsigma, \quad p_{0}, p \in \tilde{M} .
$$

The surface $\tilde{S}$ is the double surface of a nonorientable, regular minimal surface in $\mathbf{R}^{3}$ if and only if

$$
\left\{\begin{array}{lll}
\text { (i) } & g(\tilde{I}(p))=-1 / \overline{g(p)}, & p \in \tilde{M}, \text { and } \\
\text { (ii) } & \tilde{I}^{*}(\omega)=-g^{2} \omega, & \omega=f(z) d z,
\end{array}\right.
$$

for some antiholomorphic involution $\tilde{I}: \tilde{M} \rightarrow \tilde{M}$ without fixed points.

Received by the editors October 31, 1985.

1980 Mathematics Subject Classification (1985 Revision). Primary 53A10; Secondary 30A68. 
COROLLARY 1.3. Let $\tilde{S}$ be an orientable, regular, connected minimal surface in $\mathbf{R}^{4}$ given by $\tilde{X}: \tilde{M} \rightarrow \mathbf{R}^{4}$,

$$
\tilde{X}(p)=\operatorname{Re} \int_{p_{0}}^{p}\left(1+g_{1} g_{2}, i\left(1-g_{1} g_{2}\right), g_{1}-g_{2},-i\left(g_{1}+g_{2}\right)\right) \omega, \quad p_{0}, p \in \tilde{M}
$$

Then, $\tilde{S}$ is the double surface of a nonorientable minimal surface in $\mathbf{R}^{4}$ if and only if, for some antiholomorphic involution $\tilde{I}$ on $\tilde{M}$ without fixed points,

$$
\left\{\begin{array}{lll}
\text { (i) } & g_{k}(\tilde{I}(p))=-1 / \overline{g_{k}(p)}, & k=1,2, \text { and } \\
\text { (ii) } & \tilde{I}^{*} \omega=\overline{g_{1} g_{2} \omega}, & \omega=f(z) d z
\end{array}\right.
$$

If $C(\tilde{S})$ is the total curvature of $\tilde{S}$, we can define the total curvature of $S$ by

$$
C(S)=C(\tilde{S}) / 2
$$

In what follows, the double surface $\tilde{S}$ will always be connected, complete and of finite total curvature $C(\tilde{S})=2 \pi \tilde{m}$. From Chern and Osserman's theorem [1] we have $\tilde{M}$ conformally equivalent to a compact Riemann surface of genus $\tilde{\gamma}$ punctured at $\tilde{r}=2 r$ points $\left\{p_{1}, p_{2}, \ldots, p_{r}, q_{1}, \ldots, q_{r}\right\}$, with $\tilde{I}\left(p_{j}\right)=q_{j}, \tilde{I}\left(p_{j}\right)$ being the extension of $\tilde{I}$ to $p_{j}, 1 \leq j \leq r$. The function $\tilde{\phi}$ of (1.1) has poles of order $m_{j}$ at $p_{j}$ and $q_{j}$, and

$$
\tilde{m}-2 \sum_{j=1}^{r} m_{j}=2 \tilde{\gamma}-2 .
$$

The Chern and Osserman inequality for nonorientable minimal surfaces is

$$
C(S) \leq 2 \pi(\chi(M)-r)
$$

with $\chi(M)$ the Euler characteristic of $M$.

A nonorientable version of Gackstatter's theorem [2] gives an estimate for the dimension of a nonorientable, complete, minimal surface $S$ with total curvature $C(S)=-2 \pi m, r$ ends and genus $\gamma$ :

$$
\operatorname{Dim}(S) \leq 2 m-2 \gamma-r+3 .
$$

We have

PROPOSITION 1.4. The total curvature of a nonorientable, regular, complete minimal surface is at most $-4 \pi$.

ProOF. From (1.7) and the fact that $\operatorname{Dim} S \geq 3,2 m \geq 4$.

2. Genus one nonorientable complete minimal surfaces in $\mathbf{R}^{n}$. A characterization of the complete minimal surfaces of finite total curvature and genus 
one can be set up combining Theorem 1.1 and Hoffman and Osserman's theorem [5]:

THEOREM 2.1. Let $\tilde{M}$ be the complex plane minus $2 r-1$ points $\left\{0, z_{1}, \ldots, z_{r-1}\right.$, $\left.-1 / \bar{z}_{1}, \ldots,-1 / \bar{z}_{r-1}\right\}$. Let the complex vector $\phi(\zeta)$ be of the form

$$
\phi(\varsigma)=F(\varsigma)\left(p_{1}(\varsigma), \ldots, p_{n}(\varsigma)\right)
$$

with

$$
F(\varsigma)=\left(\varsigma^{\nu_{0}} \prod_{k=1}^{r-1}\left(\varsigma-z_{k}\right)^{\nu_{k}}\left(\varsigma+\frac{1}{\bar{z}_{k}}\right)^{\nu_{k}}\right)^{-1}
$$

$p_{j}(\zeta)$ satisfying

(i) $p_{j}(\zeta)$ is a polynomial,

(ii) the maximum degree of $p_{j}$ is $2 m$,

(iii) the $p_{j}$ 's have no common factor,

(iv) $\sum_{j=1}^{n} p_{j}^{2} \equiv 0$,

(v) $(-1)^{m+1} \bar{\zeta}^{2 m} p_{j}(-1 / \bar{\zeta})=\overline{p_{j}(\zeta)}, 1 \leq j \leq m$.

Suppose that the $\nu_{k}$ 's satisfy $\sum_{k=0}^{r-1} \nu_{k}=m+1, \nu_{k} \geq 2,0 \leq k \leq r-1$ and $\operatorname{Re} \int_{\gamma} \phi=0$, for any simple closed curve $\gamma$ in $\tilde{M}$. Then $\tilde{X}(p)=\operatorname{Re} \int_{p_{0}}^{p} \phi(\zeta) d \zeta$ defines a double minimal surface associated to a nonorientable regular complete minimal surface in $\mathbf{R}^{n}$ of genus one, $r$ ends and total curvature $-2 \pi m$.

Conversely, every genus one nonorientable minimal surface in $\mathbf{R}^{n}$ admits a representation as mentioned above.

PROOF. The double surface associated to a genus one nonorientable minimal surface is conformally equivalent to the complex plane $\hat{\mathbf{C}}$ minus $2 r$ points with the involution $\tilde{I}: \mathbf{C} \rightarrow \mathbf{C}$ given by $\tilde{I}(z)=-1 / \bar{z}$, that is,

$$
\tilde{M}=\mathbf{C}-\left\{0, z_{1}, \ldots, z_{r-1},-1 / \bar{z}_{1}, \ldots,-1 / \bar{z}_{r-1}\right\} .
$$

From Hoffman and Osserman's result, $\phi=F \cdot\left(p_{1}, \ldots, p_{n}\right), p_{j}$ polynomials satisfying (i)-(iv).

The map $\tilde{X}: \tilde{M} \rightarrow \mathbf{R}^{n}$ defines a double surface if and only if $\tilde{I}^{*}\left(\phi_{j}(\zeta) d \zeta\right)=$ $\overline{\phi_{j}(\zeta) d \zeta}, 1 \leq j \leq n$; from this fact and (1.5), we have $\sum_{k=0}^{r-1} \nu_{k}=m+1$ and (v).

We study initially surfaces whose total curvature is the upper bound given by Proposition 1.4.

THEOREM 2.2. Let $S$ be a nonorientable, regular, complete minimal surface of finite total curvature $-4 \pi$. Then $S$ is a minimal immersion of the projective plane minus one point and lies fully in $\mathbf{R}^{4}$; any two such minimal surfaces are similar.

Proof. From (1.5), and (1.6) and (1.7), using $\chi(M)=2-\gamma-r$, we have $\gamma=1, r=1$ and $m_{1}=3$. The double surface has genus zero and two ends; thus, by Theorem 2.1 , it can be given by $\tilde{X}: C-\{0\} \rightarrow \mathbf{R}^{n}$,

$$
\tilde{X}_{j}(p)=\operatorname{Re} \int_{p_{0}}^{p} \phi_{j}(\varsigma) d \varsigma, \quad p_{0}, p \in \tilde{M}=\mathbf{C}-\{0\}, \phi_{j}(\varsigma)=p_{j}(\varsigma) / \varsigma^{3},
$$

$p_{j}$ satisfying conditions (i)-(v). 
Setting $p_{j}(\zeta)=a_{j} \varsigma^{4}+b_{j} \varsigma^{3}+c_{j} \varsigma^{2}+d_{j} \zeta+e_{j}$, we can verify that $a_{j}=-e_{j}$, $b_{j}=\bar{d}_{j}, c_{j}=0$ and that $A_{1}=\left(\operatorname{Re} a_{1}, \ldots, \operatorname{Re} a_{n}\right), A_{2}=\left(\operatorname{Im} a_{1}, \ldots, \operatorname{Im} a_{n}\right), B_{1}=$ $\left(\operatorname{Re} b_{1}, \ldots, \operatorname{Re} b_{n}\right)$ and $B_{2}=\left(\operatorname{Im} b_{1}, \ldots, \operatorname{Im} b_{n}\right)$ are real orthogonal vectors of the same length.

Thus, $A=(1, i, 0,0)$ and $B=(0,0,1, i)$ give a solution in $\mathbf{R}^{4}$; two solutions $\tilde{X}$ and $\tilde{Y}$ are such that $\tilde{Y}=\lambda M \tilde{X}+X_{0}$, with $\lambda \in \mathbf{R}$ and $M$ a real orthogonal matrix.

Immediately, we have Meeks' theorem:

COROLlaRY 2.3. The total curvature of a nonorientable, regular, complete minimal surface in $\mathbf{R}^{3}$ is at most $-6 \pi$.

The total curvature of an orientable minimal surface in $\mathbf{R}^{3}$ is of the form $C(\tilde{S})=$ $-4 \pi \cdot \operatorname{degree}(g)$, with $g$ the meromorphic function of the Weierstrass representation. Thus, for nonorientable minimal surfaces,

$$
C(S)=-2 \pi \cdot \text { degree }(g)
$$

with $g: \tilde{M} \rightarrow \mathbf{C}$ the function of the Weierstrass representation of the double surface $\tilde{S}$, which is a rational function when it has finite degree and the genus of $\tilde{M}$ is zero.

The next result is very important for the construction of examples:

PROPOSITION 2.4. Let $g$ be a rational complex function satisfying $g(-1 / \bar{z})=$ $-1 / \overline{g(z)}$. Then, the degree of $g$ is odd and $g$ is of the form

$$
g(z)=c z^{\alpha} \frac{\prod_{j=1}^{m}\left(z-a_{j}\right)}{\prod_{j=1}^{m}\left(z+1 / \bar{a}_{j}\right)}, \quad \text { with }|c| \prod_{j=1}^{m}\left|a_{j}\right|=1 .
$$

PROOF. Let $g$ be a rational function, and let

$$
g(z)=c z^{\alpha} \frac{\prod_{j=1}^{m}\left(z-a_{j}\right)}{\prod_{k=1}^{n}\left(z-b_{k}\right)}, \quad a_{j} \neq b_{k} \text { for any } j, k, a_{j} \neq 0, b_{k} \neq 0
$$

where the factors appear with multiplicities. From the condition

$$
g(-1 / \bar{z})=-1 / \overline{g(z)}
$$

with some calculations, we complete the proof.

PROPOSITION 2.5. The total curvature of a nonorientable regular complete minimal surface in $\mathbf{R}^{3}$ of genus one is of the form $C(S)=-2 \pi m, m$ odd, $m \geq 3$.

ProOF. This follows immediately from Proposition 2.4.

Meeks [6] has proved that there exists a unique nonorientable regular complete minimal surface in $\mathbf{R}^{3}$ of total curvature $-6 \pi$. We extend Meeks' surface in the sense of

THEOREM 2.6. There exists a nonorientable regular complete minimal surface in $\mathbf{R}^{3}$ of genus one, one end and total curvature $-2 \pi m$, for any $m$ odd, $m \geq 3$.

PROOF. With straightforward calculations, one can show that

$$
f(z)=i(z+1)^{2} / z^{m+1} \text { and } g(z)=z^{m-1}(z-1) /(z+1)
$$

are the functions of the Weierstrass representation of a double minimal surface $\tilde{S}$; that is, the map

$$
\tilde{X}(p)=\operatorname{Re} \int_{p_{0}}^{p} \frac{f}{2}\left(1-g^{2}, i\left(1+g^{2}\right), 2 g\right) d \zeta, \quad p_{0}, p \in \mathbf{C}-\{0\},
$$


satisfies (i)-(v) of Theorem 2.1. The surfaces obtained are regular and complete.

REMARKS. 1. If $n=3$ the surface of Theorem 2.6 is exactly Meeks' surface.

2. The surface is an infinite Moebius band with $(m-1) / 2$ twists and the image of $|z|=1$ is a circle centered at $(0,-2 /(m-1), 0)$ with radius $2 /(m-1)$ covered $(m-1)$ times.

3. The normals to the surface along $|z|=1$ have the third component $\left.N_{3}\right|_{|z|=1}=$ $\left.\operatorname{Re}(z)\right|_{|z|=1}$.

The Chern-Osserman inequality for nonorientable minimal surfaces of total curvature $-6 \pi$ gives us the relation $\gamma+2 r \leq 5$; hence, if $\gamma=1$, we may have $r=1$ or $r=2$. Meeks has proved that the case $r=2, C=-6 \pi$ cannot occur. Thus, we have

PROPOSITION 2.7. The total curvature of a nonorientable regular complete minimal surface in $\mathbf{R}^{3}$ of genus one and two ends is at most $-10 \pi$.

For $C=-10 \pi$, we have

THEOREM 2.8. There exists a nonorientable regular complete minimal surface in $\mathbf{R}^{3}$ of genus one, two ends and total curvature $-10 \pi$.

PROOF. The double surface associated to such a surface will be an orientable surface of genus zero, four ends with total curvature $-20 \pi$ and, by Theorem 2.1 , it may be given by

$$
\tilde{X}: \mathbf{C}-\{0,1,-1\} \rightarrow \mathbf{R}^{3}, \quad \tilde{I}: \mathbf{C} \rightarrow \mathbf{C}, \quad \tilde{I}(z)=-1 / \bar{z} .
$$

Let $f(z)=a\left(\bar{b}^{2} z^{2}-1\right)^{2} / z^{2}(z-1)^{4}(z+1)^{4}$ and $g(z)=\alpha \bar{b}^{2} z^{3}\left(z^{2}-b^{2}\right) /\left(\bar{b}^{2} z^{2}-1\right)$; $f$ and $g$ are the functions of the Weierstrass representation of a double minimal surface if and only if

$$
|\alpha||b|^{2}=1 \text { and } a=-\bar{a} \bar{\alpha} b^{4} \text { or } a \alpha \bar{b}^{2}=-\bar{a} \bar{\alpha} b^{2} .
$$

The completeness and regularity can easily be verified.

To choose $\alpha, a$ and $b$ such that

$$
\operatorname{Re} \int_{\gamma} \frac{f(x)}{2}\left(1-g^{2}(z), i\left(1+g^{2}(z)\right), 2 g(z)\right) d z=\overrightarrow{0}
$$

for any closed curve $\gamma$ in $\mathbf{C}-\{0,1,-1\}$, we observe that, by Cauchy's integral formula, for simple closed curves $\gamma_{0}$ and $\gamma_{1}$ around $z=0$ and $z=1$, respectively,

$$
\int_{\gamma_{0}} \frac{p_{j}(\zeta) d \zeta}{\varsigma^{2}(\zeta-1)^{4}(\zeta+1)^{4}}=2 \pi i p_{j}^{\prime}(0)
$$

and

$$
\begin{aligned}
& \int_{\gamma_{1}} \frac{p_{j}(\varsigma) d \varsigma}{\varsigma^{2}(\varsigma-1)^{4}(\varsigma+1)^{4}}=\frac{2 \pi i}{3 !} \cdot \frac{1}{16}\left[p_{j}^{\prime \prime \prime}(1)-12 p_{j}^{\prime \prime}(1)+57 p_{j}^{\prime}(1)-105 p_{j}(1)\right] \\
& \text { Calling } R_{j}=p_{j}^{\prime \prime \prime}(1)-12 p_{j}^{\prime \prime}(1)+57 p_{j}^{\prime}-105 p_{j}(1), j=1,2,3 \text {, we have } \\
& R_{1}=-105(a-\bar{a})+30\left(a \bar{b}^{2}-\bar{a} b^{2}\right)+3\left(a \bar{b}^{4}-\bar{a} b^{4}\right), \\
& R_{2}=i\left[-105(a+\bar{a})+30\left(a \bar{b}^{2}+\bar{a} b^{2}\right)+3\left(a \bar{b}^{4}+\bar{a} b^{4}\right)\right] \text {, } \\
& R_{3}=0 \text {. }
\end{aligned}
$$


Thus, $\operatorname{Re}\left[2 \pi i R_{j}\right]=0, j=1,2$ if $-105 a+30 a \bar{b}^{2}+3 a \bar{b}^{4}=0$.

For each $b, \alpha=-1 / \bar{b}^{2}, a=i$ satisfy $(2.2)$ and

$$
\tilde{X}(p)=\operatorname{Re} \int_{p_{0}}^{p} \frac{f}{2}\left(1-g^{2}, i\left(1+g^{2}\right), 2 g\right) d \zeta
$$

gives the double surface.

We investigate the problem of the regular minimal surfaces in $\mathbf{R}^{3}$ of finite total curvature, genus one and three ends, and, we have

THEOREM 2.9. There exists a nonorientable regular complete minimal surface in $\mathbf{R}^{3}$ of genus one, three ends and total curvature $-14 \pi$.

Proof. We construct the double surface $\tilde{S}$ defined by $\tilde{X}: \mathbf{C}-\{0,1,-1, i,-i\} \rightarrow$ $\mathbf{R}^{3}$ with the functions $f$ and $g$ of the Weierstrass representation (1.2) given by $g(z)=a \bar{b}^{4} z^{3}\left(z^{4}-b^{4}\right) /\left(\bar{b}^{4} z^{4}-1\right)$ and $f(z)=\alpha\left(\bar{b}^{4} z^{4}-1\right)^{2} / z^{2}\left(z^{2}-1\right)^{3}\left(z^{2}+1\right)^{3}$. The functions $f$ and $g$ satisfy (1.2) if and only if

$$
|a||b|^{4}=1, \quad \alpha=\bar{\alpha} \bar{a}^{2} b^{8} .
$$

With some calculations, we can verify that the real parts of the integrals

$$
\int_{\gamma} \frac{p_{j}(z) d z}{z^{2}\left(z^{4}-1\right)^{3}}
$$

where $\gamma$ is a simple closed curve, are null for any $j, j=1,2,3$, provided $b$ satisfies the following condition: $3 b^{8}+10 b^{4}-45=0$. Thus, $b^{4} \in \mathbf{R}$.

Choosing $a=1 / b^{4}$ and $\alpha=1$ the conditions (2.3) are verified. The completeness and regularity can be easily checked.

In $\mathbf{R}^{4}$, exploring the properties of the functions $g_{1}, g_{2}$ of the representation (1.3), we have

THEOREM 2.10. For any integer $m, m \geq 2$, there exists a nonorientable regular complete minimal surface $S$ in $\mathbf{R}^{4}$ of genus one, one end and total curvature $-2 \pi m$.

Proof. The double surface $\tilde{S}$ associated to $S$ has total curvature $-4 \pi m=$ $-2 \pi \tilde{m}, \tilde{m}=2 m$, genus zero and two ends. We set the functions $f, g_{1}, g_{2}$ in the representation (1.3) by $f(z)=z^{m-1}, g_{1}(z)=\varepsilon_{1} / z^{k_{1}}, g_{2}(z)=\varepsilon_{2} / z^{k_{2}}$, with $\tilde{m}=$ $2 m=k_{1}+k_{2}, k_{1}, k_{2}$ odd, $k_{1}, k_{2} \geq 1$, and $\tilde{M}=\mathrm{C}-\{0\}$.

The functions $f, g_{1}$ and $g_{2}$ satisfy (i) and (ii) of Corollary 1.3, if and only if

$$
\left|\varepsilon_{1}\right|=\left|\varepsilon_{2}\right|=1, \quad \varepsilon_{1} \varepsilon_{2}=(-1)^{m-1} \text {. }
$$
that

To verify that $\operatorname{Re} \int_{\gamma} \phi(\varsigma) d \zeta=\overrightarrow{0}$, for any closed curve in $\mathbf{C}-\{0\}$, we observe

$$
\begin{aligned}
& \phi_{1}(\varsigma)=f\left(1+g_{1} g_{2}\right)=z^{m-1}+\varepsilon_{1} \varepsilon_{2} / z^{m+1} \\
& \phi_{2}(\varsigma)=i f\left(1-g_{1} g_{2}\right)=i\left(z^{m-1}-\varepsilon_{1} \varepsilon_{2} / z^{m+1}\right) \\
& \phi_{3}(\varsigma)=f\left(g_{1}-g_{2}\right)=\left(\varepsilon_{1} z^{k_{2}}-\varepsilon_{2} z^{k_{1}}\right) / z^{m+1} \\
& \phi_{4}(\varsigma)=-i f\left(g_{1}+g_{2}\right)=-i\left(\varepsilon_{1} z^{k_{2}}+\varepsilon_{2} z^{k_{1}}\right) / z^{m+1}
\end{aligned}
$$


and rewriting $k_{1}=m-n, k_{2}=m+n, n \geq 1$, we have $\int_{\gamma} \phi_{j}=0, j=1, \ldots, 4$. Thus, the double surface $\tilde{S}$ is defined by

$$
\tilde{X}(p)=\operatorname{Re} \int_{p_{0}}^{p} \phi(\zeta) d \zeta, \quad p_{0}, p \in \mathbf{C}-\{0\} .
$$

For $\gamma=1, r=1$, the dimension of $S$ satisfies $\operatorname{Dim}(S) \leq 2 m$. We will show that this upper bound is sharp.

THEOREM 2.11. For any integer $m, m \geq 2$, there exists a nonorientable regular complete minimal surface $S$ in $\mathbf{R}^{2 m}$ of genus one, one end and total curvature $-2 \pi m$ that lies fully in $\mathbf{R}^{2 m}$.

Proof. The double surface $\tilde{S}$ associated to $S$, by Theorem 2.1 , is given by $\tilde{X}: \mathbf{C}-\{0\} \rightarrow \mathbf{R}^{n}, \tilde{X}(p)=\operatorname{Re} \int_{p_{0}}^{p} \phi(\varsigma) d \varsigma$, with $\phi_{j}(\varsigma)=p_{j}(\varsigma) / \varsigma^{m+1}, p_{j}(\varsigma)$ satisfying (i) $-(\mathrm{v})$.

Particularly, condition (v) is equivalent to

$$
(-1)^{m+1} \bar{\zeta}^{2 m} p_{j}(-1 / \bar{\zeta})=\overline{p_{j}(\zeta)}, \quad 1 \leq j \leq n
$$

Taking $p_{j}(\varsigma)=a_{0}^{j}+a_{1}^{j} \varsigma+\cdots+a_{2 m-1}^{j} \varsigma^{2 m-1}+a_{2 m}^{j} \varsigma^{2 m}$ in (2.5) we obtain

$$
(-1)^{m+1}\left(a_{0}^{j} \bar{\zeta}^{2 m}-a_{1}^{j} \bar{\zeta}^{2 m-1}+\cdots+a_{2 m}^{j}\right)=\left(\bar{a}_{2 m}^{j} \bar{\zeta}^{2 m}+\bar{a}_{2 m-1}^{j} \bar{\zeta}^{2 m-1}+\cdots+\bar{a}_{0}^{j}\right) \text {. }
$$

Then, there are two possibilities:

(a) $(m+1)$ even: $a_{0}^{j}=\bar{a}_{2 m}^{j},-a_{1}^{j}=\bar{a}_{2 m-1}^{j}, \ldots, a_{m-1}^{j}=\bar{a}_{m+1}^{j},-a_{m}^{j}=\bar{a}_{m}^{j}$,

(b) $(m+1)$ odd: $a_{0}^{j}=-\bar{a}_{2 m}^{j}, a_{1}^{j}=\bar{a}_{2 m-1}^{j}, \ldots, a_{m-1}^{j}=\bar{a}_{m+1}^{j}, a_{m}^{j}=-\bar{a}_{m}^{j}$.

The integrals $\int_{\gamma} \phi_{j}(\zeta) d \zeta, \gamma$ a closed curve in $\mathbf{C}-\{0\}$, should have null real part; therefore, $\operatorname{Re}\left\{2 \pi i \cdot a_{m}^{j}\right\}=0,1 \leq j \leq n$, that is $\operatorname{Im} a_{m}^{j}=0,1 \leq j \leq n$. Comparing with the last condition in (a) or (b), we have $a_{m}^{j}=0,1 \leq j \leq n$.

To determine solutions in $\mathbf{R}^{n}$, we should find complex vectors $A_{k}=\left(a_{k}^{1}, \ldots, a_{k}^{n}\right)$, $0 \leq k \leq m-1$, such that the corresponding polynomials satisfy $\sum_{j=1}^{n} p_{j}^{2}(\varsigma) \equiv 0$.

It is easy to verify that $A_{0}=(1, i, 0, \ldots, 0), A_{1}=(0,0,1, i, 0, \ldots, 0), \ldots$, $A_{m-1}=(0, \ldots, 0,1, i)$ give a solution in $\mathbf{C}^{2 m}$ if $m$ is even and $A_{0}=(1, i, 0, \ldots, 0)$, $A_{2}=(0,0,1, i, 0, \ldots, 0), \ldots, A_{m-2}=(0,0, \ldots, 0, \sqrt{2}, i \sqrt{2}, 0,0), A_{m-1}=(0,0, \ldots$, $0,1, i)$ give a solution if $m$ is odd.

With these results, we have partial answers for the problem of existence of nonorientable, regular, minimal surfaces in $\mathbf{R}^{3}$ and $\mathbf{R}^{n}$ posed by Nitsche $[\mathbf{7}, 1965]$. We do not know, for instance, an example of a genus two nonorientable minimal surface in $\mathbf{R}^{3}$, that is, a minimal Klein bottle in $\mathbf{R}^{3}$.

\section{REFERENCES}

1. S. S. Chern and R. Osserman, Complete minimal surfaces in Euclidean n-space, J. Analyse Math. 19 (1967), 15-30.

2. F. Gackstatter, Über die Dimension eine minimalfläche und zur Ungleichung von St. Cohn-Vossen, Arch. Rational Mech. Anal. 61 (1976), 141-152.

3. L. Henneberg, Über salche minimalfläche, welche eine vorgeschriebene ebene curve sur geodätishen line haben, Doctoral Dissertation, Eidgenössisches Polythechikum, Zurich, 1875.

4. __ Über diejenige minimalfläche, welche die Neil'sche Paralee zur ebenen geodätischen line hat, Vierteljschr Natuforsch, Ges. Zürich 21 (1876), 66-70. 
5. D. A. Hoffman and R. Osserman, The geometry of the generalized Gauss map, Mem. Amer. Math. Soc. No. 28 (1980).

6. W. H. Meeks III, The classification of complete minimal surfaces with total curvature greater than $-8 \pi$, Duke Math. J. 48 (1981), 523-535.

7. J. C. C. Nitsche, On new results in the theory of minimal surfaces, Bull. Amer. Math. Soc. 71 (1965), 195-270.

8. M. E. G. Oliveira, Superfícies mínimas não-orientáveis no $R^{n}$, Tese de Doutoramento-IME-USP, 1984.

9. R. Osserman, A survey of minimal surfaces, Van Nostrand Reinhold, New York, 1969.

10. __ Global properties of minimal surfaces in $E^{3}$ and $E^{n}$, Ann. of Math. (2) 80 (1964), 340-364.

11. M. Spivak, A comprehensive introduction to differential geometry, Vol. 3, Publish or Perish, Boston, Mass., 1975.

Instituto de Matemática e Estatística, Universidade de SÃo Paulo, CaiXa Postal 20.570 (Agência Iguatemi), SÃo PaUlo, Brazil 